\title{
The Effect of Various Parameters of Solution Methodology on a Flexible Integrated Supply Chain Model
}

\author{
Elham Behmanesh $\mathbb{D}^{1}$ and Jürgen Pannek $\mathbb{D}^{2,3}$ \\ ${ }^{1}$ International Graduate School for Dynamics in Logistics, University of Bremen, Bremen, Germany \\ ${ }^{2}$ Faculty of Production Engineering, University of Bremen, Bremen, Germany \\ ${ }^{3}$ BIBA-Bremer Institut für Produktion und Logistik GmbH, Bremen, Germany
}

Correspondence should be addressed to Elham Behmanesh; beh@biba.uni-bremen.de

Received 8 March 2017; Accepted 18 February 2018; Published 3 April 2018

Academic Editor: Giuseppe Carlo Marano

Copyright (C) 2018 Elham Behmanesh and Jürgen Pannek. This is an open access article distributed under the Creative Commons Attribution License, which permits unrestricted use, distribution, and reproduction in any medium, provided the original work is properly cited.

\begin{abstract}
A successful supply chain must be able to operate at the lowest cost while providing the best customer service as well as environmental protection. As industrial players are under pressure but mostly unprepared to take back products after their usage, logistics network design becomes an even more important issue. To allow for a maximum of flexibility and efficiency, we consider an integrated design of the forward/reverse logistics network using full delivery graph. We apply a Memetic Algorithm with a novel population generation to find a near optimal solution for large size problems. The effect of different parameters on the behavior of the proposed Metaheuristic Algorithm is investigated. Using the experimental work to find the best parameters for this problem is the outlook of these researches.
\end{abstract}

\section{Introduction}

A supply chain is a network of suppliers, manufacturers, warehouses, distributions, and retailers. It is organized to produce and distribute products, and the respective design issue is to determine the number, location, and capacity of the facilities as well as the quantity of the flow between them [1]. The aim of supply chain design is minimization of total cost $[1,2]$ or profit maximization [3] while satisfying customer requirements. Each supply chain network involves decisions on three different levels, operational, tactical, and strategic, which summarize short, mid, and long term decisions, respectively. As long term strategic decisions take priority over tactical and operational ones, network configuration comes first in any supply chain network design and needs to be optimized for the long-lasting efficient operation of entire supply chain.

In the open-loop supply chain, which is the traditional (forward) system, products do not return to their source. Within the closed-loop (forward/reverse) supply chain, the returned products are collected by respective centers to reuse the materials or products by the original manufacturers or to dispose of them in specific disposal centers. Considering this extension of the supply chain becomes more relevant as in the last decade governmental legislation forced firms to collect, recover, and recycle, for example, all electronic goods in Europe, Japan, China, and many parts of US and Canada as well as safe disposal of their end-of-life products [4]. As a consequence, closed-loop supply chain has become a pressing topic for supply chain partners. Although some firms such as HP, Dell, General Motors, Kodak, Caterpillar, and Xerox concentrated on reverse logistics and have obtained significant successes $[5,6]$, most logistics networks are not equipped to handle reverse products.

Environmental factors are not the only reason that spurs researchers or manufacturers to consider closed-loop logistic networks. The reverse activity represents an economic added value that can be obtained by processing the returned products. Indeed, one ton of electronic computer waste contains more gold than 17 tons of material extracted from a gold mine [7]. In most of the past researches, the design of forward and reverse logistics networks is considered separately, which 


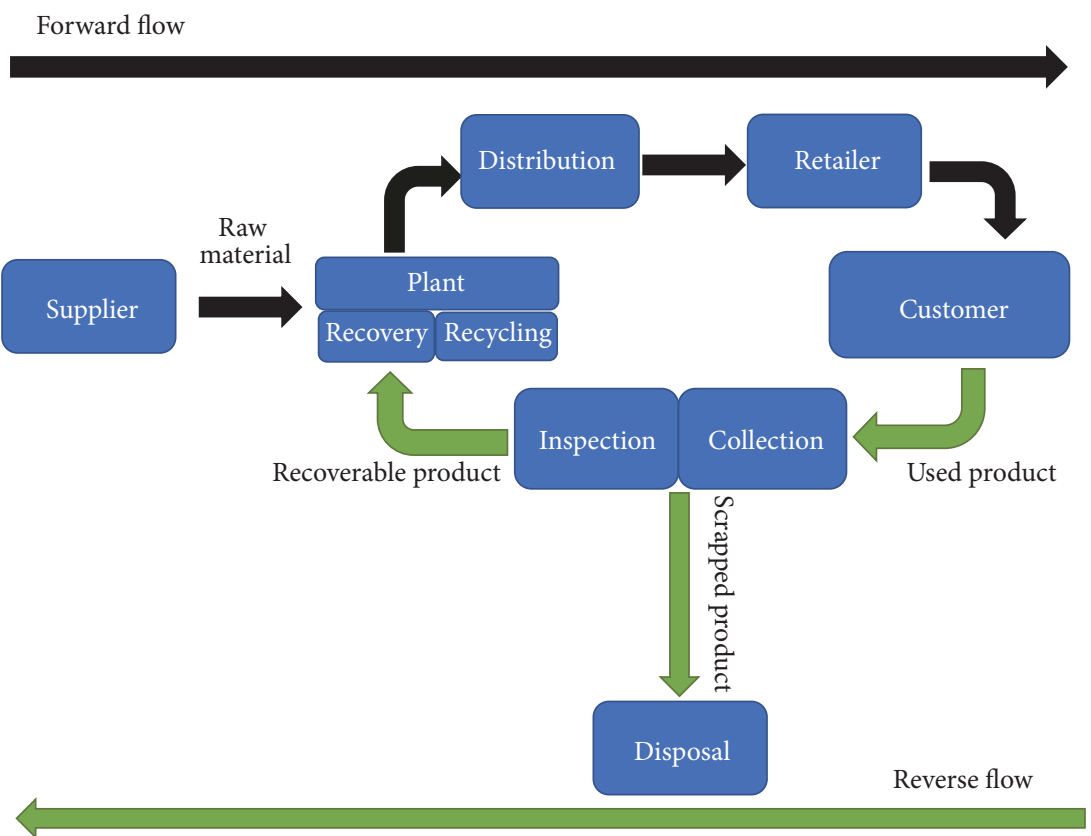

FIGURE 1: Framework of the proposed closed-loop supply chain network.

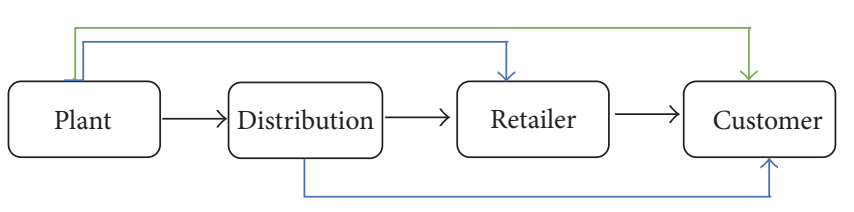

FIGURE 2: Delivery graph for the proposed flexible supply chain network.

may result in a suboptimal solution. Consequently, both parts should be considered simultaneously [8], which leads us to a closed-loop supply chain network (cf. Figure 1 for a sketch). In this figure, the classical (forward) and reverse supply chains are presented by black and green lines, respectively. In the returned flow, returned products are collected by collection centers and after inspection the recoverable products are shipped to the plant for further operations while scrapped products are transferred to disposal centers for safe disposal.

Fast and on time delivery of products with a great accuracy plays an important role in customer satisfaction. The ability of supply chain to satisfy the customer's expected delivery time is called "supply chain responsiveness" $[9,10]$. Some previous researchers have proposed models to optimize the supply chain network with regard to both cost efficiency and responsiveness simultaneously $[9,11]$. However, most of these researches limited themselves to only consider shipment between consecutive stages or just indirect shipment mechanisms $[9,11,12]$. Yet, efficiency can be improved by considering all possible delivery ways, which renders the problem to be more complex (cf. Figure 2 for a sketch of the delivery graph).

Based on the aforementioned descriptions, within this paper we consider an integrated forward/reverse multistage, single product, and single period logistics network, which is enriched by a full delivery graph representing a NP-hard mixed integer linear programming model. To cope with the NP-hard problems in supply chain networks, many heuristic algorithms [1, 12-14] and metaheuristics such as Genetic Algorithm [11, 15-19], Simulated Annealing [20, 21], Tabu Search [8, 22], Memetic Algorithm [9], and Scatter Search [12] have been proposed. However, there is still a critical need in this area to increase the efficiency of solution approaches, especially, when the complexity of the model increases (cf., e.g., [23]). To address the latter, in this work we propose a Memetic Algorithm with a novel idea to generate population. We particularly focus on the impact of different parameters such as size of population, crossover rate, and local search iteration as well as number of iterations without improvement on convergence and solution properties of the proposed algorithm.

The remainder of the paper is structured as follows: after reviewing related literature in Section 2, the mathematical model of the comprehensive multistage closed-loop supply chain network is presented as a mixed integer linear programming (MILP) model in Section 3. In Section 4 we present the solution methodology to solve the MILP model. We have numerical setting and analysis of our results with regard to several parameters in Sections 5 and 6, and last with conclusion and some suggestions for future research Section 7 closes our study.

\section{Literature Review and Problem Definition}

There are some studies regarding the effect of different parameters of metaheuristic algorithms applied for logistics networks problems. Lee and Dong [8] proposed a MILP 
model, which is able to manage the forward and reverse distributions at the same time for end-off lease computer products. Regarding the solution methodology, they used a Memetic Algorithm based on Tabu Search. They observed that the maximum number of iterations does not make a significant impact on the performance of the proposed heuristics in all the five presented test problems. From their result, it is clear that about 80 percent of the progress towards the optimum is obtained within 20 iterations. They also presented some results with different number of iterations within a neighborhood search. Since this work was focusing on a specific case study for end-of-lease computer products recovery, the lower bound of total cost in the distribution network was available and adapted for the purpose of solution comparison. The computation times for the heuristic presented in this study increase reasonably as the problem size increases. Syarif et al. [24] proposed a MILP formulation for a fixed charge and multistage transportation problem. A spanning tree based Genetic Algorithm is applied to solve this problem. Based on their results, the crossover rate is suggested to be equal to 0.4 , the mutation rate is fixed as 0.2 , and the population size is set to 100 . To have more information about the proposed algorithm, they divided each test problem into three numerical experiments by giving different population size. They conclude that since the search space for large size problems is so huge, it is very important to set the experiment with reasonable population size and maximum generation in order to ensure increased chances for good results.

Wang and Hsu [17] presented a closed-loop model with a spanning tree based Genetic Algorithm. In this study, the crossover rate is fixed as 0.4 and the mutation rate as 0.2 , and for the population they considered different numbers to observe its influence with respect to problem size. Moreover, several conditions are settled for number of generations, computing time, and fitness convergence. Fitness convergence appears when all chromosomes in the population have the same fitness value. The authors stop the evolutionary process in their implementation when the number of iterations without improvement in fitness value is 10 . At the same time, they impose the maximal number of generations to be 750 as another stopping criterion. From simulations, the authors realized that in the proposed algorithm increasing the population size improves accuracy for large size problems only slightly while the required computation times are huge. Therefore, they concluded that using large population size for large size test problems is not sufficient.

Pishvaee et al. [20] focused on a MILP model to minimize the cost including transportation cost as well as operation cost in a reverse logistics network. A Simulated Annealing method with a new neighborhood search is applied to find a near optimal solution. In this study, four different cooling rates are imposed. From simulations, the authors concluded that the standard deviation is increasing for increased sizes of test problems. In order to evaluate the performance of the proposed algorithm, a fixed ratio of the standard deviation is used. To assess the different cooling rates, several statistical tests are presented. The results showed that just two cooling rates are acceptable with respect to their computation time and gaps. The extended version of two-stage transportation problem is developed by Gen et al. [26] to minimize total cost including opening cost of distribution centers and shipping cost from plant to distribution center as well as distribution center to customers. As solution methodology, a priority based Genetic Algorithm is presented using a new crossover method called Weight Mapping Crossover (WMX). Simulations were carried out in two stages. In the first stage, the authors investigated the effect of different combinations of crossover and mutation operators. The aim of this stage was to find the best combination among the 8 possibilities given by 4 crossover and 2 mutation methods. In the second stage, each test problem is divided into three numerical experiments to assess the effect of population size and number of generations on the performance of the proposed Genetic Algorithm. In addition, to show the performance of the priority based Genetic Algorithm, another chromosome representation called spanning tree based Genetic Algorithm using Prüfer number is considered. Statistical analysis using different population sizes and numbers of generations showed the improved performance of the priority based Genetic Algorithm considering the average performance. Yet, it requires more computation time in comparison to the spanning tree based Genetic Algorithm. Regarding the second stage, the results showed an improvement in quality of solution by increasing the population size as well as number of generations. The authors mentioned that a tradeoff between solution quality and computation time exists. Khorshidian et al. [27] applied a Genetic Algorithm for justin-time single machine scheduling. The model in this study is nonlinear with integer variables. To assess the effectiveness of chosen parameters of the proposed algorithm, several test problems were conducted. They considered four crossover rate levels, four mutation rates, three population sizes, and two maximal numbers of generations. A Taguchi analysis is applied to find the best parameters for this specific problem. The obtained values were set as default values for further evaluation. A multiresponse problem was presented by Zandieh et al. [28] within a desirability function framework. The main focus of this research is to determine the best parameters values for a Genetic Algorithm, a Tabu Search, and a Simulated Annealing method. Simulations were carried out into two stages. In the first stage, the authors specified the appropriate level for each parameter of each algorithm using Taguchi analysis. In the second stage, the parameters with significant impact on robustness of the proposed algorithm were determined.

Based on the above review, we can summarize the following:

(i) Considering the effect of all parameters of solution methodology is typically ignored and most papers focus on just one or two factors.

(ii) The general influence of parameters on the overall behavior of an algorithm is typically done in a case by case manner. 
The problem addressed in this work is an integrated design of forward and reverse logistics enriched by three different delivery paths for a seven-stage, single period, single product closed-loop supply chain network. The proposed model presents a general network and covers the previously described cases in the literature with less complexity. Furthermore, considering a full delivery graph in forward flow allows us to solve the conflicting goals profit and responsiveness which otherwise may cause a greater cost [9]. In order to increase the efficiency of the proposed algorithm, some information about the parameters applied in Memetic Algorithm such as number of iterations, local search iteration, population size, and crossover rate in format of a parameter analysis is required. To this end, studying the effect of these parameters is the main contribution of this study.

\section{Description for Integrated Forward/Reverse Logistics Network}

In this section we support the presentation of the proposed mathematical model by considering the general model area of our problem. In order to achieve this particular aim, $G=$ $(N, E)$ is considered as a digraph. Here, $N$ denotes the set of all nodes and $E$ the set of all edges in the proposed flexible integrated forward/reverse logistics network. We denote the cost for node $i \in N$ by $c_{i}$ and the unit transportation cost on edge $(i, j) \in E$ by $c_{i j}$. The respective decision variables are given by $y_{i}$ and $x_{i j}$, where $y_{i} \in\{0,1\}$ and $x_{i j} \in \mathbb{N}_{0}$. These variables indicate whether a stage $i \in N$ is used and which quantity is transported from nodes $i$ to $j$, respectively. The minimization of transportation and operation cost of the proposed flexible integrated forward/reverse logistics network is considered in pursuance of determining the optimal capacity of each node as well as of the flow between them. For this purpose, the following mixed integer minimization problem is considered:

$$
\begin{array}{ll}
\min _{x_{i j}, y_{i}} & \sum_{(i, j) \in E} c_{i j} x_{i j}+\sum_{i \in N} c_{i} y_{i}, \\
\text { s.t. } & \sum_{(i, j) \in E} a_{i} x_{i j} \leqslant b_{i} y_{i} \\
& x_{i j} \geq 0, y_{i} \in\{0,1\} .
\end{array}
$$

More specifically, we consider a seven-stage closed-loop supply chain network, which consists of five stages in forward flow including suppliers $S$, plants $P$, distribution centers Dc, retailers $R$, and customers $C$ and two stages in reverse flow featuring collection/inspection centers Co and disposal centers Di (cf. Figure 3 for a schematic sketch). Since establishing several facilities at the same location is advantageous with regard to costs due to sharing equipment and infrastructures [29], we consider a hybrid manufacturing-recovery-recycling facility as well as a hybrid collection-inspection facility. To adapt problem (1), the following assumptions are imposed:

(i) The set of nodes is resembled by $N=S \cup P \cup \operatorname{Dc} \cup R \cup$ $C \cup \mathrm{Co} \cup \mathrm{Di}$. (ii) The set of edges is given by $E=(S \times P) \cup(P \times \mathrm{Dc}) \cup$ $(P \times R) \cup(P \times C) \cup(\mathrm{Dc} \times R) \cup(\mathrm{Dc} \times C) \cup(R \times$ $C) \cup(C \times \mathrm{Co}) \cup(\mathrm{Co} \times \mathrm{Di}) \cup(\mathrm{Co} \times P)$. There is a full graph between plant and customer in forward flow. In reverse flow, normal delivery is considered. Moreover, no connections between facilities of the same stage are allowed.

(iii) The number of facilities per stage and their capacities are fixed and known.

(iv) The demands of each customer are deterministic and must be satisfied. All cost parameters (fixed and variable) are known in advance.

(v) The price is completely equal for all facilities in the same stage; that is, customers have no preference.

(vi) The transportation rates are perfect and there are no storages. Moreover, the return rate $p_{j}^{\text {return }}$ and the recovery and disposal rates $p_{j}^{\text {disposal }}$ and $\left(1-p_{j}^{\text {disposal }}\right)$ are fixed. All returned products must be collected.

(vii) The inspection cost per item is included in the collection cost.

(viii) The recoverable products are returned to the same plant and the unrecyclable products are transferred to the disposal center for safe disposal.

(ix) The quality of recycled and raw materials is assumed to be identical. For each plant, choosing the raw material form collection/inspection center has priority over suppliers.

In terms of the mentioned notation, the cost function, the sign, and the integer conditions remain identical. The constraints in (1) are given by

$$
\sum_{(i, j) \in E} x_{i j} \leq \begin{cases}b_{i} & \forall i \in S \\ b_{i} y_{i} & \forall i \in N \backslash\{S \cup C\} .\end{cases}
$$

Furthermore, in-flow and out-flow in each node must be identical except for the return fraction $p^{\text {return }}$ the disposal fraction $p^{\text {disposal }}$ of products. Combined, this resembles the constraint

$$
\begin{aligned}
& \sum_{(j, k) \in E} x_{j k} \\
& = \begin{cases}\sum_{(i, j) \in E} x_{i j} & \forall j \in N \backslash\{C \cup \mathrm{Co}\} \\
p_{j}^{\text {return }} \sum_{(i, j) \in E} x_{i j} & \forall j \in C \\
p_{j}^{\text {disposal }} \sum_{(i, j) \in \mathrm{CoxDi}} x_{i j} & \forall j \in \text { Co } \\
\left(1-p_{j}^{\text {disposal }}\right) \sum_{(i, j) \in \mathrm{Co} \times P} x_{i j} & \forall j \in \mathrm{Co} .\end{cases}
\end{aligned}
$$

Last, all demands of customers must be satisfied, which induces the constraint

$$
\sum_{(i, j) \in E} x_{i j}=b_{j} \quad \forall j \in C .
$$




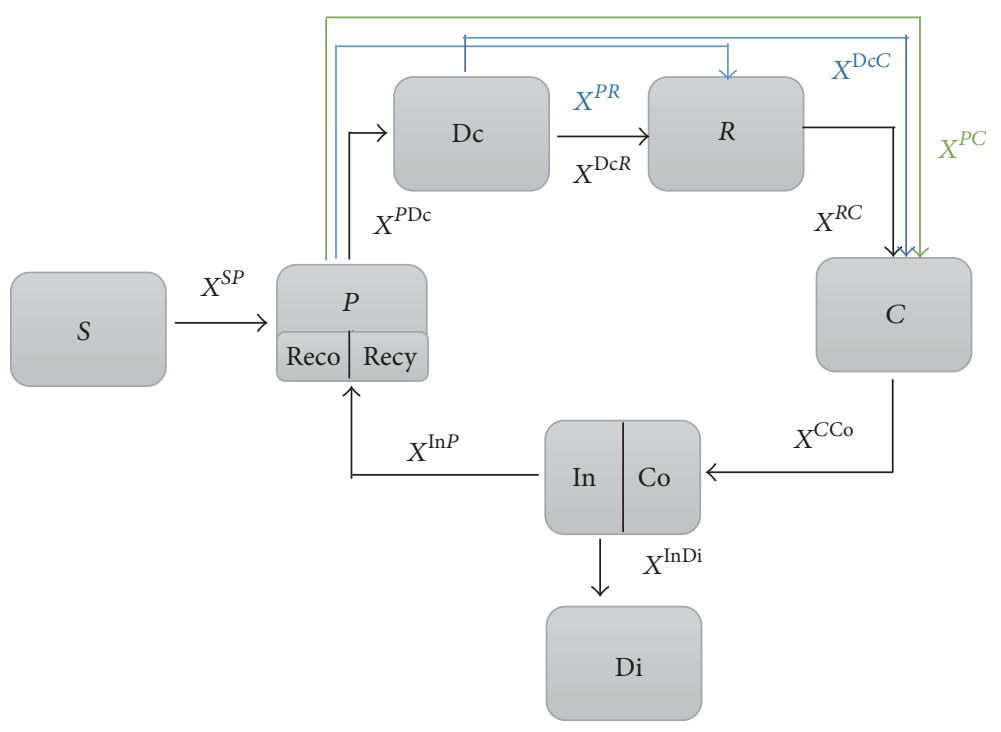

FIGURE 3: Underlying structure of MILP.

\section{Solution Approach}

Since our network design problem represents an NP-hard problem [16, 30-32], mixed integer linear programming cannot derive a suitable solution for large scale problems in acceptable time. Memetic Algorithms, however, belong to class of metaheuristic algorithms, which have been applied successfully for the proposed model and similar cases [25, 33-37]. A complete explanation has been presented in [25] regarding the procedure of initialization by extended random path-base direct encoding, two-point crossover, and local search as well as the Memetic Algorithm applied in this study.

According the aforementioned components, we consider the procedure displayed in Figure 4 for solving our problem. In this flowchart, we have included different colors, showing the main focus of this study. Note that as we apply only one crossover and search step before selecting the next generation, our method belongs to the class of steady state MA.

\section{Analysis of the Extended MA}

In general, there are two important factors to analyze each Metaheuristic Algorithm: diversification and intensification. Diversification is required to investigate the new area in the search space, which is covered by crossover and a high population size in our approach. Intensification, on the other hand, seeks to improve the solution, which in the proposed case is considered by a local search. For each Metaheuristic Algorithm both are necessary but contradictory in solving an optimization problem. By having a balance between them, results will be higher efficiency and better effect.

To validate our approach, we consider LINGO [25] as a benchmark to assess the behavior and performance of the proposed MA in terms of accuracy of the obtained solution under different values of the related effective parameters. Within this study, one particular condition is selected and changing any other parameter is considered till the effect of each parameter is recorded and analyzed.

5.1. Design of Experiments. Experiments for the Memetic Algorithms have shown that there exist two major impact sources affecting the performance of Memetic Algorithms [17], that is, the chromosome representation and the memetic operators. Here, we utilize the chromosome representation from [25] and analyze the effect of various parameters on the memetic operators regarding both performance and computing time. We particularly focus on

(i) number of iterations,

(ii) population size,

(iii) number of local search iterations,

(iv) crossover rate.

5.2. Termination Conditions. Traditionally [38], termination conditions for Genetic and Memetic Algorithms include bounds on the number of generations or the evaluations of the fitness function or may be triggered if the chance of achieving significant changes in the next generations is excessively low. For the first two alternatives, we require some knowledge about the problem. In contrast to that, the third one does not require such knowledge and may be implemented, for example, by imposing a bound on the number of iterations without improvement.

\section{Computation Results}

Since the logistics network framework in this study differs from previous studies, we generated six small and medium size test problems with $128,209,234,468,1006$, and 1780 decisions variables (cf. Table 1 ), to assess the effect of parameters mentioned in Section 5 on the developed MA. Other parameters of the logistics network were generated randomly 


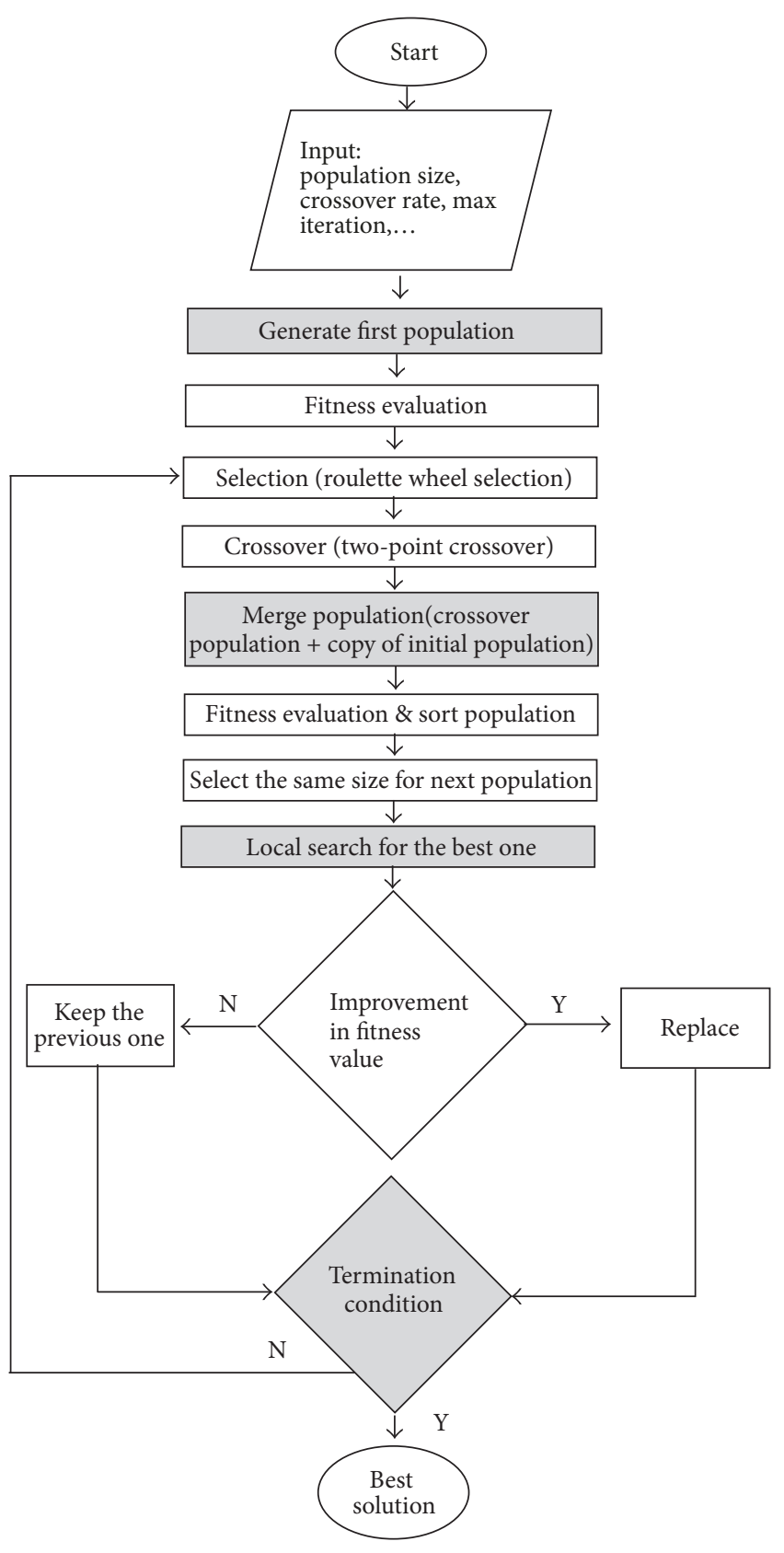

FIGURE 4: The flow diagram of proposed MA.

using uniform distributions (cf. Table 2). To show the performance of proposed MA, we employed the Branch-andBound Algorithm from LINGO15 to solve the optimization problem. Respective results are included in Table 1. Each test problem has been solved 20 times to test the robustness of the method.

Our implementation was written in MATLAB R2015b and run on the PC with Intel ${ }^{\circledR}$ Core $^{\mathrm{TM}}$ i5 $2.40 \mathrm{GHz}$ with $12 \mathrm{~GB}$ RAM.

6.1. Number of Iterations without Improvement. During our simulations, we observed that a specific maximal number
TABLE 1: Settings of test problems.

\begin{tabular}{lcccccc}
\hline Problem & 1 & 2 & 3 & 4 & 5 & 6 \\
\hline Supplier & 2 & 2 & 2 & 2 & 3 & 4 \\
Plant & 2 & 4 & 3 & 4 & 6 & 8 \\
Distribution & 5 & 6 & 8 & 10 & 15 & 20 \\
Retailer & 8 & 10 & 9 & 16 & 24 & 32 \\
Customer & 2 & 2 & 3 & 4 & 6 & 8 \\
Collection/inspection center & 2 & 2 & 3 & 4 & 6 & 8 \\
Disposal & 1 & 1 & 2 & 2 & 2 & 4 \\
Total number of facilities & 22 & 27 & 30 & 42 & 62 & 84 \\
Total number of possible routes & 110 & 218 & 294 & 432 & 966 & 1728 \\
\hline Solution by LINGO & 2905 & 2345 & 2335 & 1160 & 4100 & 11365 \\
\hline
\end{tabular}

TABle 2: Parameters values used in the test problems [25].

\begin{tabular}{lc}
\hline Parameters & Range \\
\hline$b_{j}, j \in S$ & Uniform $(200,1100)$ \\
$b_{j}, j \in P$ & Uniform $(100,1000)$ \\
$b_{j}, j \in \mathrm{Dc}$ & Uniform $(50,900)$ \\
$b_{j}, j \in R$ & Uniform $(50,850)$ \\
$b_{j}, j \in D$ & Uniform $(100,500)$ \\
$b_{j}, j \in \mathrm{Co}$ & Uniform $(20,100)$ \\
$b_{j}, j \in \mathrm{Di}$ & Uniform $(20,100)$ \\
$p_{j}^{\text {return }}$ & $10 \%$ \\
$p_{j}^{\text {disposal }}$ & $50 \%$ \\
$c_{i j}$ & Uniform $(1,3)$ \\
$c_{j}, j \in P$ & Uniform $(100,2500)$ \\
$c_{j}, j \in \mathrm{Dc}$ & Uniform $(100,2100)$ \\
$c_{j}, j \in R$ & Uniform $(100,400)$ \\
$c_{j}, j \in \mathrm{Co}$ & Uniform $(100,500)$ \\
$c_{j}, j \in \mathrm{Di}$ & Uniform $(50,400)$ \\
\hline
\end{tabular}

of iterations without improvement cannot be chosen simultaneously for all test problems. Hence, in a first step we determined the number of iterations without improvement for each test problem specifically. To this end, we consider several instances and selected the largest candidate for which significant changes may be achieved.

To assess the effect of the termination criterion on the proposed MA, we imposed a maximum iteration number of 200 and varied the bound on the number of iterations without improvement for the test problems. As the criterion appears to be depending on the size of the problems, we considered different ranges for the bound (cf. Figures 5(a) and 5(b) and Table 3 ). We reported the minimum cost, maximum cost, and average cost from 20 independent runs, along with the average CPU time.

These bar charts allow us to identify the critical range for our braking criterion. For example, given test problem 1, choosing max number of iterations without improvement to be 10 is advantageous. From a performance point of view, we consider this bound to be set to 6 to assess the effect of other parameters. 


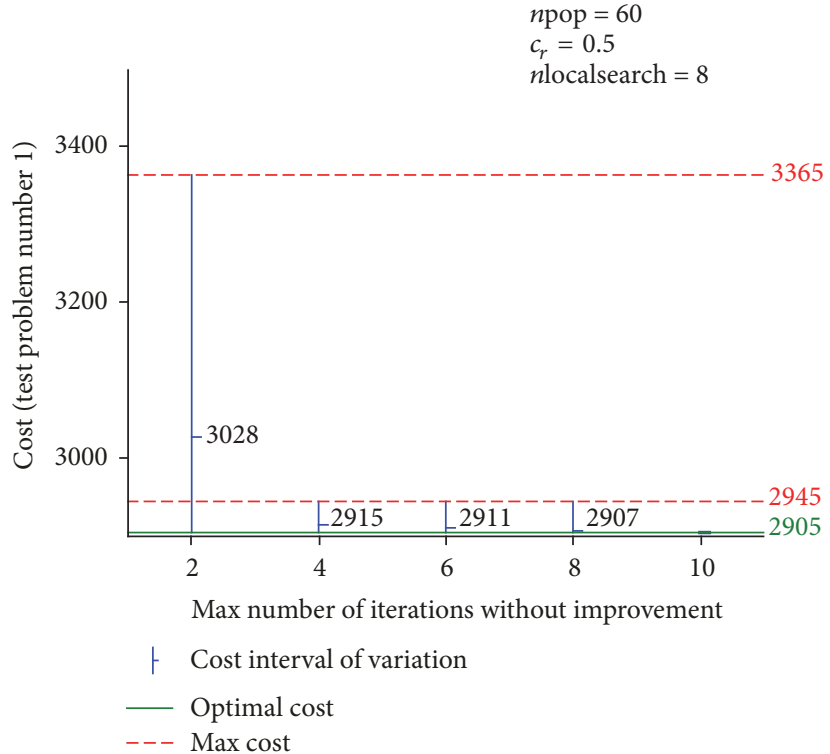

(a) Test problem number 1

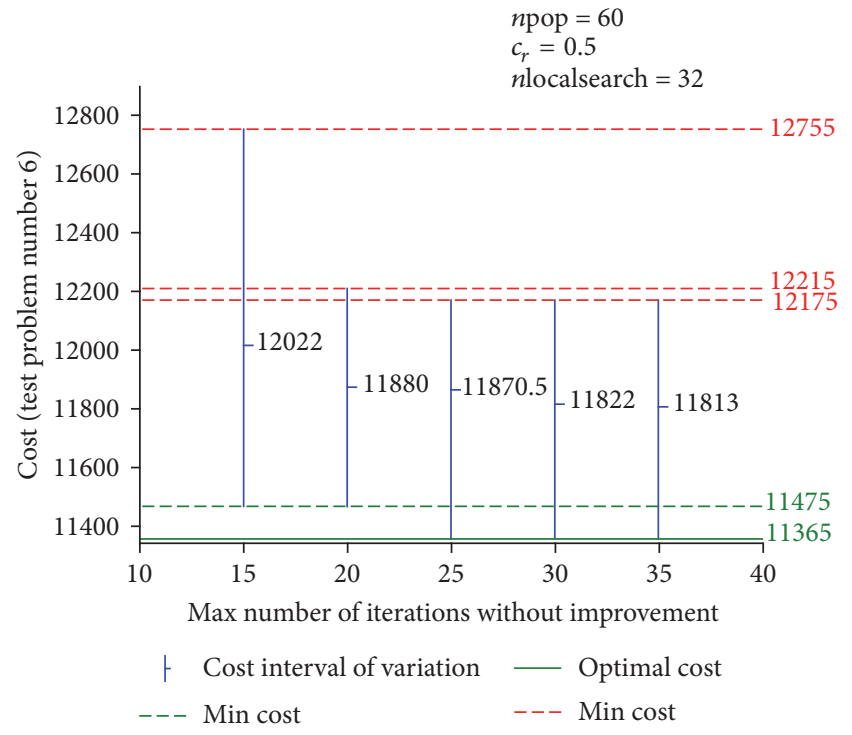

(b) Test problem number 6

Figure 5: Gap of the proposed MA for different bounds on the number of iterations without improvement.

TABLE 3: Results of the proposed MA under different maximal number of iterations without improvement.

\begin{tabular}{|c|c|c|c|}
\hline \multicolumn{4}{|c|}{ Test problem number 2} \\
\hline Max number of iterations without improvement & Min cost & Max cost & Ave cost \\
\hline 4 & 2345 & 3545 & 2456 \\
\hline 6 & 2345 & 3145 & 2451 \\
\hline 8 & 2345 & 2885 & 2381 \\
\hline 10 & 2345 & 2885 & 2378 \\
\hline 12 & 2345 & 2885 & 2378 \\
\hline \multicolumn{4}{|c|}{ Test problem number 3} \\
\hline Max number of iterations without improvement & Min cost & Max cost & Ave cost \\
\hline 6 & 2335 & 2815 & 2460 \\
\hline 8 & 2335 & 2695 & 2418 \\
\hline 10 & 2335 & 2535 & 2360 \\
\hline 12 & 2335 & 2535 & 2360 \\
\hline 14 & 2335 & 2535 & 2345 \\
\hline \multicolumn{4}{|c|}{ Test problem number 4} \\
\hline Max number of iterations without improvement & Min cost & Max cost & Ave cost \\
\hline 5 & 1160 & 1860 & 1223.5 \\
\hline 10 & 1160 & 1840 & 1208 \\
\hline 15 & 1160 & 1730 & 1195.5 \\
\hline 20 & 1160 & 1660 & 1188.5 \\
\hline 25 & 1160 & 1360 & 1184 \\
\hline \multicolumn{4}{|c|}{ Test problem number 5} \\
\hline Max number of iterations without improvement & Min cost & Max cost & Ave cost \\
\hline 10 & 4100 & 5490 & 4325 \\
\hline 15 & 4100 & 5490 & 4265 \\
\hline 20 & 4100 & 4690 & 4218 \\
\hline 25 & 4100 & 4690 & 4196 \\
\hline 30 & 4100 & 4320 & 4182.6 \\
\hline
\end{tabular}




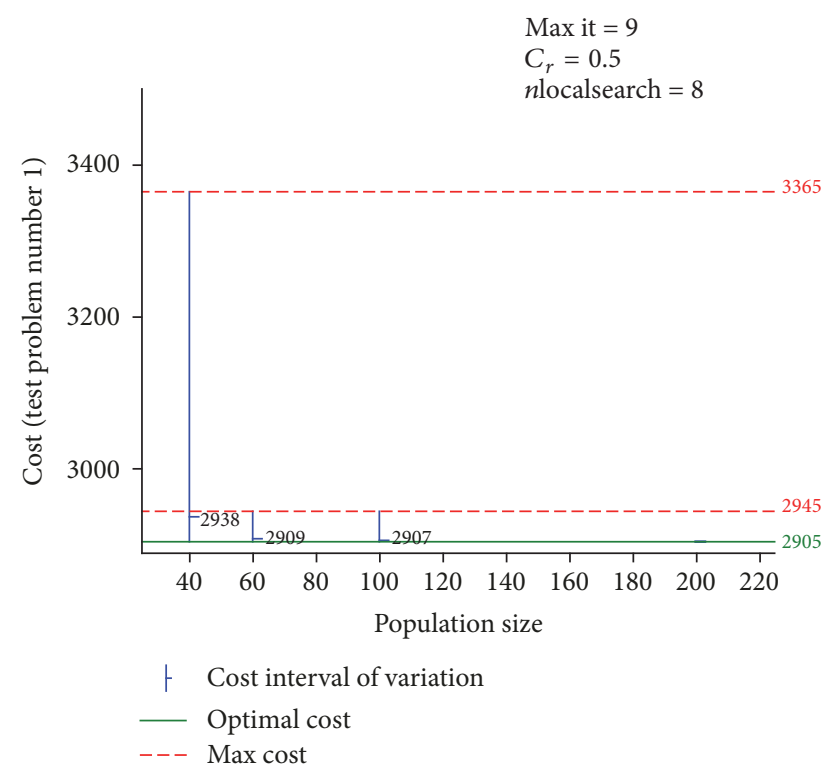

(a) Problem number 1

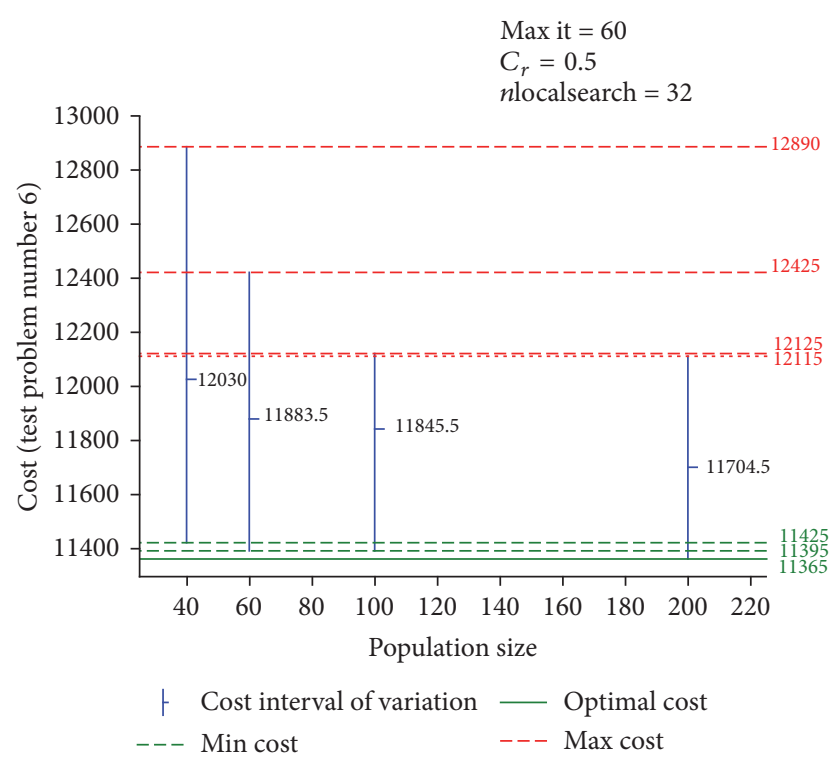

(b) Problem number 6

FiguRE 6: Gap of the proposed MA for different population sizes.

TABLE 4: Results of the proposed MA for different population sizes.

\begin{tabular}{lcccc}
\hline Test problem & 2 & 3 & 4 & 5 \\
\hline population size & Cost & Cost & Cost & Cost \\
& Min, max, ave & Min, max, ave & Min, max, ave & Min, max, ave \\
\hline 40 & $2345,3205,2437$ & $2335,2795,2436$ & $1160,1730,1223.5$ & $4100,4720,4332$ \\
60 & $2345,2405,2375$ & $2335,2535,2370$ & $1160,1530,1208.5$ & $4100,4490,4265$ \\
100 & $2345,2405,2351$ & $2335,2535,2345$ & $1160,1360,1205.5$ & $4100,4300,4252.5$ \\
200 & $2345,2345,2345$ & $2335,2335,2335$ & $1160,1230,1177.5$ & $4100,4190,4167.5$ \\
\hline
\end{tabular}

Additionally, we computed the minimum, maximum, and average number of iterations and selected the average number of iterations to fix the number of iterations for further assessment. Note that the minimum number of iterations is not sufficient and the maximum number of iterations is too large to assess the effect of other parameters. Strictly speaking, the number of iterations should be high enough so as to obtain good results, but not too high to avoid influencing other parameters. By choosing the maximum number of iterations without improvement to be 6 , it results in the average number of iterations equal to 9 for test problem number 1 .

Similarly, 8, 14, 20, 25, and 30 are selected as the maximal number of iterations without improvement for test problem number 2 to 6 . According to these selections, we obtain $14,22,39,43$, and 60 as the average number of iterations, respectively, to assess the effect of other parameters.

6.2. Size of Population. Population size is often specified by the user and remains fixed [39]. In most of recent studies, the population size is considered as problem-independent but algorithm dependent variable is typically chosen between 50 and 100 individuals. It is found that an improper choice of the population size may lead the algorithm to less efficiency [39]. However, there is a clear relation between the population size and the convergence speed. To see the effect of population size on the proposed Memetic Algorithm, four different population size settings with 40,60,100, and 200 individuals are considered (cf. Figures 6(a) and 6(b) and Table 4). The minimum cost, maximum cost, and average cost are reported to show the robustness of the solutions obtained by the proposed Memetic Algorithm.

6.3. Number of Local Search Iterations. Based on our simulations, we observed that a predefined number of local search iterations may render the method ineffective for larger test problems. To determine the effect of number of local search iterations on the performance of the proposed MA, we imposed different bounds for the test problems according to their size (cf. Figures 7(a) and 7(b) and Table 5 for respective results). The minimum cost, maximum cost, and average cost are presented to demonstrate the upper bound and lower bound of the obtained solutions generated by the proposed Memetic Algorithm.

We increased the number of local search iterations in accordance with the size of test problems. Since the number of 


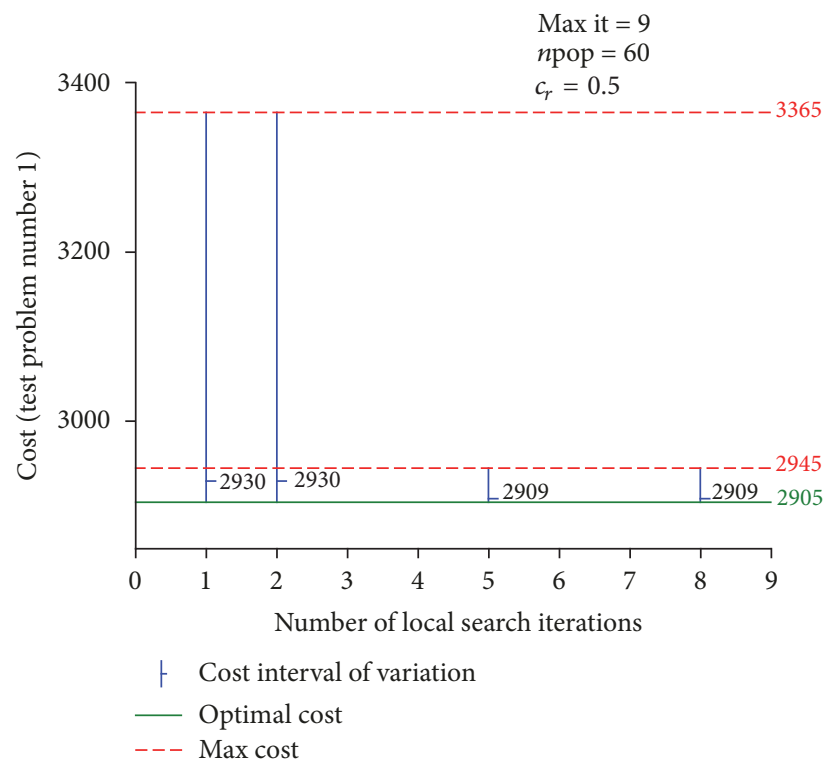

(a) Problem number 1

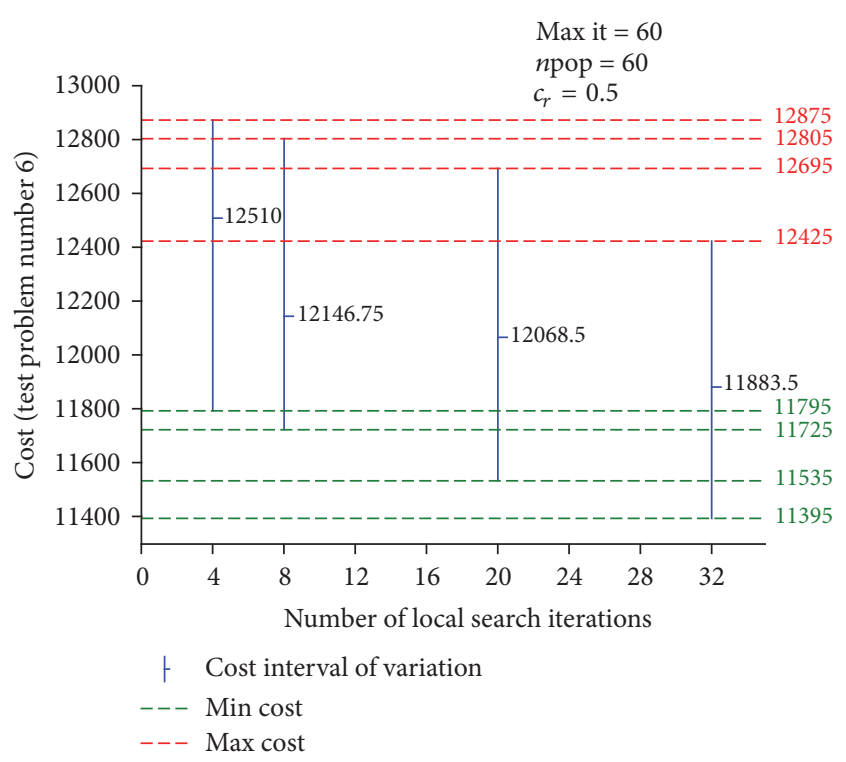

(b) Problem number 6

FIGURE 7: Gap of the proposed MA for different number of local search iterations.

TABLE 5: Results of proposed MA for different number of local search iterations.

\begin{tabular}{|c|c|c|c|}
\hline \multicolumn{4}{|c|}{ Problem number 2} \\
\hline Number of local search iterations & Min cost & Max cost & Ave cost \\
\hline 1 & 2345 & 3145 & 2456 \\
\hline 4 & 2345 & 3055 & 2451 \\
\hline 6 & 2345 & 3055 & 2381 \\
\hline 10 & 2345 & 2405 & 2378 \\
\hline \multicolumn{4}{|c|}{ Problem number 3} \\
\hline Number of local search iterations & Min cost & Max cost & Ave cost \\
\hline 2 & 2335 & 2635 & 2427 \\
\hline 3 & 2335 & 2535 & 2397 \\
\hline 8 & 2335 & 2535 & 2377 \\
\hline 9 & 2335 & 2535 & 2370 \\
\hline \multicolumn{4}{|c|}{ Problem number 4} \\
\hline Number of local search iterations & Min cost & Max cost & Ave cost \\
\hline 2 & 1160 & 1860 & 1225 \\
\hline 4 & 1160 & 1860 & 1214.5 \\
\hline 10 & 1160 & 1530 & 1211.5 \\
\hline 16 & 1160 & 1530 & 1208.5 \\
\hline \multicolumn{4}{|c|}{ Problem number 5} \\
\hline Number of local search iterations & Min cost & Max cost & Ave cost \\
\hline 2 & 4100 & 4720 & 4360 \\
\hline 6 & 4100 & 4540 & 4284.5 \\
\hline 15 & 4100 & 4490 & 4277.5 \\
\hline 24 & 4100 & 4490 & 4265 \\
\hline
\end{tabular}




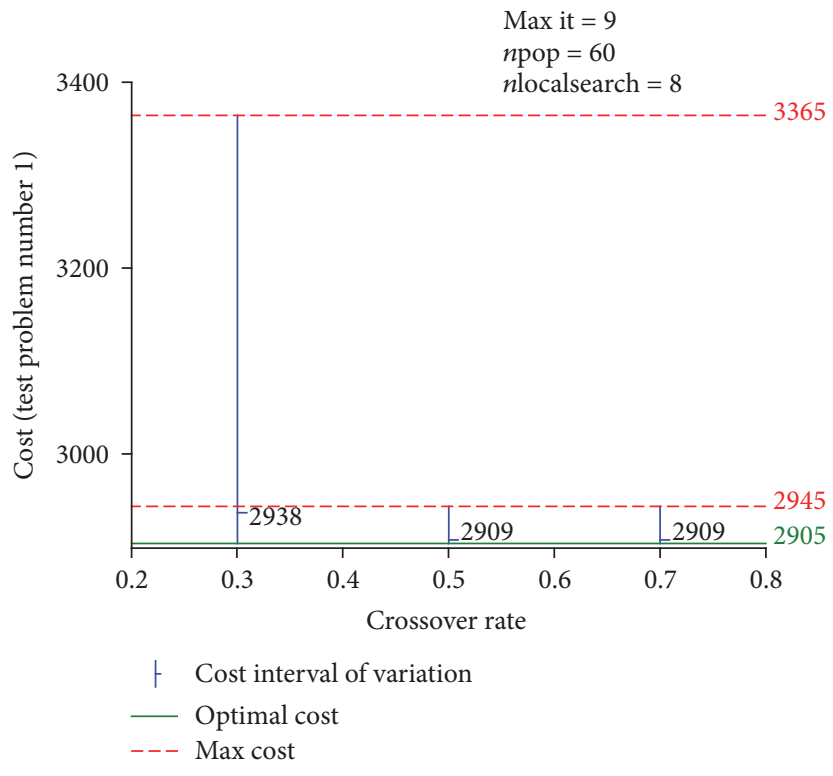

(a) Problem number 1

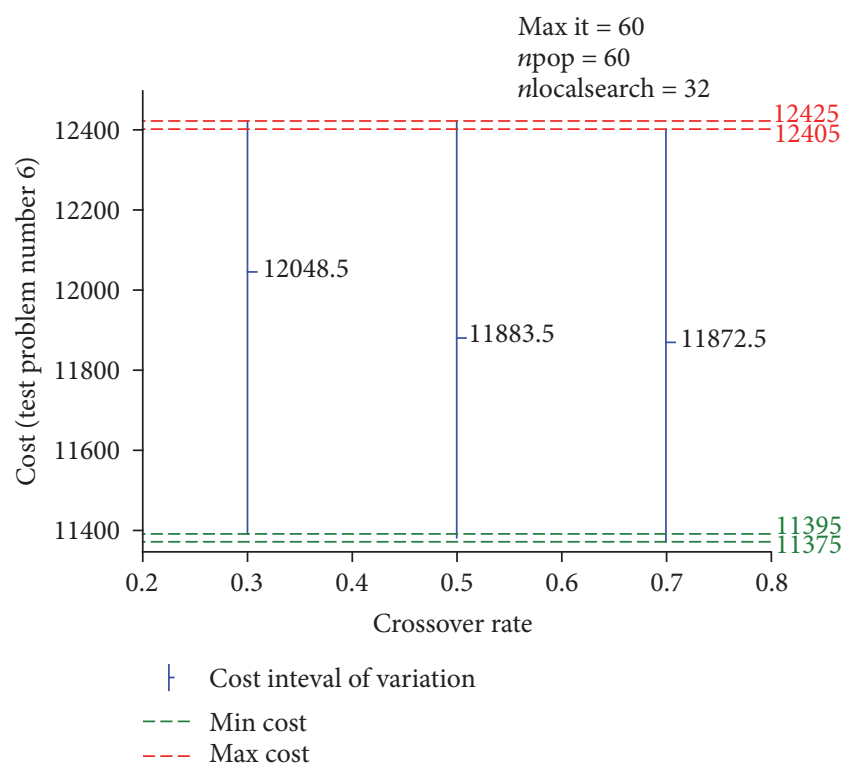

(b) Problem number 6

FIGURE 8: Gap of the proposed MA for different crossover rates.

TABLE 6: Results of proposed MA for different crossover rates.

\begin{tabular}{|c|c|c|c|c|}
\hline Problem & 2 & 3 & 4 & 5 \\
\hline \multirow{2}{*}{ Crossover rate } & Cost & Cost & Cost & Cost \\
\hline & Min, max, ave & Min, max, ave & Min, max, ave & Min, max, ave \\
\hline 0.3 & $2345,3205,2419$ & $2335,2695,2414$ & $1160,1660,1231$ & $4100,4490,4338.5$ \\
\hline 0.5 & $2345,2405,2375$ & $2335,2535,2370$ & $1160,1530,1220.5$ & $4100,4490,4265$ \\
\hline 0.7 & $2345,2405,2363$ & $2335,2535,2355$ & $1160,1360,1205.5$ & $4100,4300,4259$ \\
\hline
\end{tabular}

echelons in each stage is increased by enlarging the size of test problems, we linked the number of local search iterations to the number of disposal centers $O$, plant $J$, distribution centers $K$, and retailers $L$, respectively.

6.4. Crossover Rate. Crossover is a process of taking more than one parent chromosome to produce an offspring chromosome as a child from them. There is no single best setting to choose the crossover rate. It depends on an algorithm applied as well as other settings within the latter. Lowering the crossover rate will leave more individuals unchanged in the next generation while increasing the crossover rate leads to an increased search space of the method. To recognize the effect of crossover rate on the proposed Memetic Algorithm, three different crossover rates, $0.3,0.5$, and 0.7 , are considered as low, medium, and high crossover rates, respectively (cf. Figures 8(a) and 8(b) and Table 6). Within the information the bound of the solutions obtained by the Memetic Algorithm is available.

6.5. Result Analysis. To analyze the proposed Memetic Algorithm, the error percentage of its solution is calculated according to

$$
\text { Error percent }=\frac{\mathrm{MA}_{\text {average answer }}-\mathrm{LINGO}_{\text {answer }}}{\mathrm{LINGO}_{\text {answer }}} \cdot 100 \text {. }
$$

Figure 9(a) shows the variations in runtime for increasing the number of iterations without improvement. This behavior is similar for all six test problems and directly connected to additional iterations of the algorithm.

Figure 9(b) shows a general relation between number of iterations without improvement and error percentage for all six test problems. We observe that the error percentage is decreasing for increasing bounds. Having more iteration, let the algorithm improve the results and decrease the error percent.

As Figures 9(a) and 9(b) show, the solution gaps are shrinking for enlarged numbers of iterations without improvement. Since the latter induces higher computing times, this parameter should be increased only up to a tolerable computing time.

To assess the effect of the population size, Figures 10(a) and 10 (b) allow us to conclude that the error percentage is decreasing for enlarged populations, again at the cost of increased operation time.

The comparison between population sizes indicates that the two population sizes 60 and 100 have an acceptable performance with respect to the solution gap criterion and runtime. Although population size 200 has a high efficiency regarding solution, it does not show an acceptable running time. 


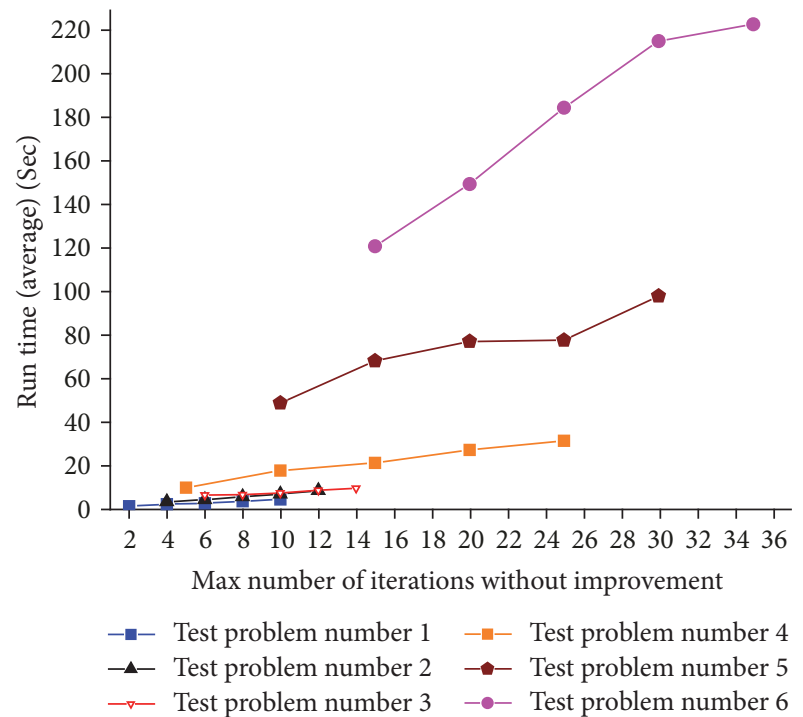

(a) Runtime of the proposed MA for different number of iterations without improvement

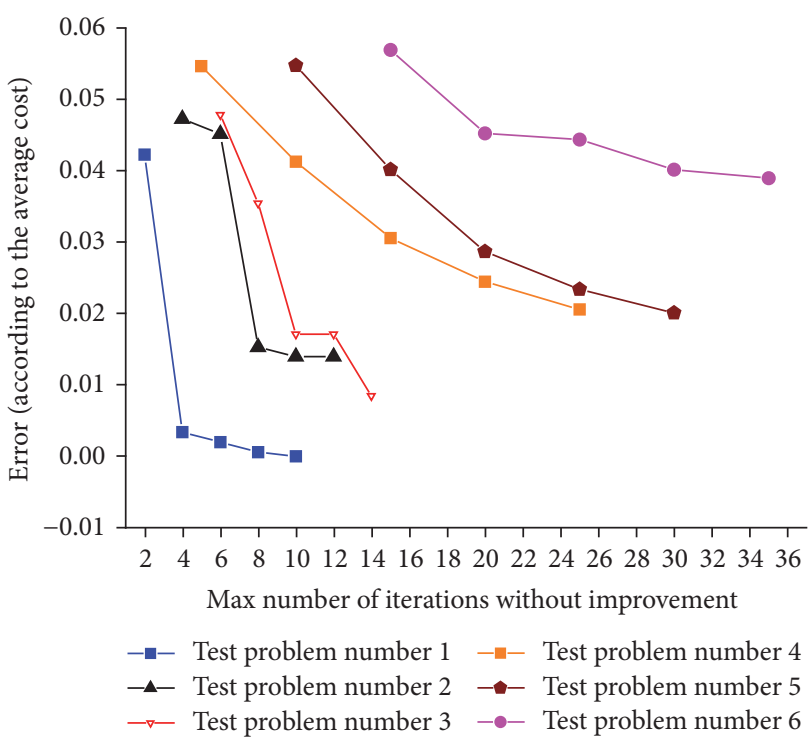

(b) Gap of the proposed MA for different number of iterations without improvement

FIgURE 9: Effect of different numbers of iterations without improvement.

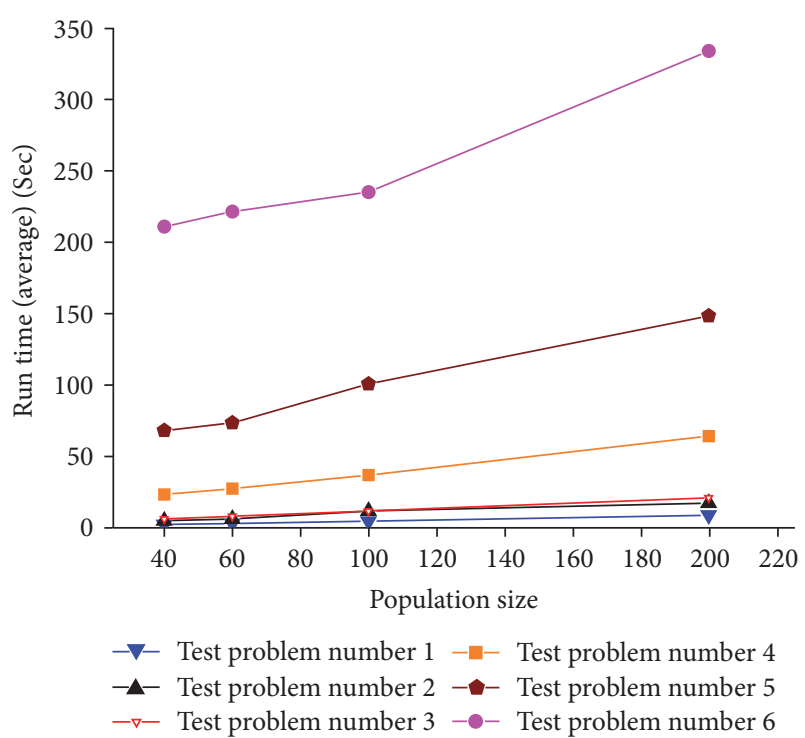

(a) Runtime of the proposed MA for different population size

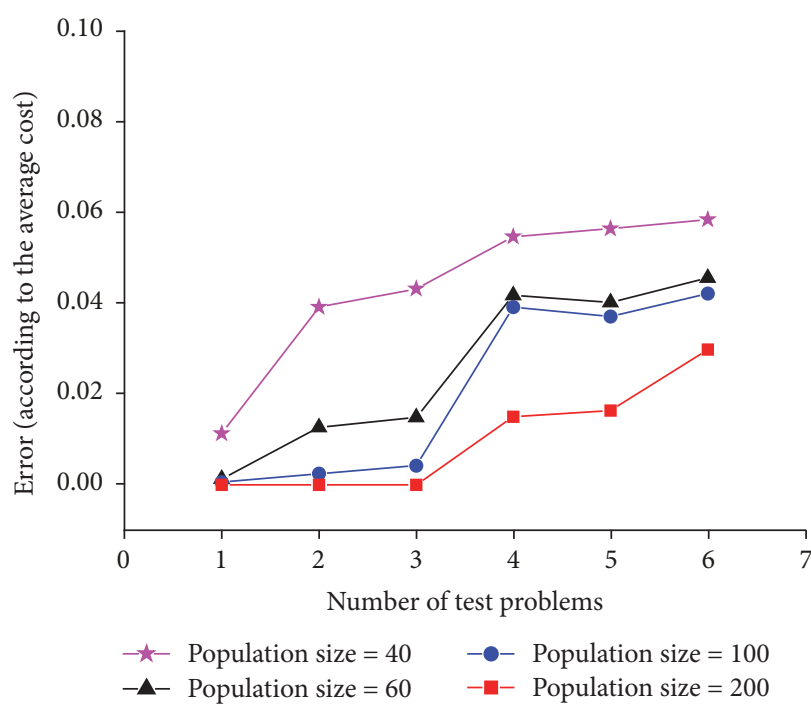

(b) Gap of the proposed MA for different population sizes

FIGURE 10: Effect of population sizes.

Regarding the effect of number of local search iterations, from Figures 11(a) and 11(b) we observe a general decrease in the error percentage by increasing the local search iteration parameter as well as a general growth in runtime. The increased computation requirements are due to the additional local search steps.

Because of the beneficial impact of this parameter on the quality of the solution, we can improve the latter at the cost of computing time.

From Figures 12(a) and 12(b) we observe that increasing the crossover rate causes the error percentage to decrease and the runtime to increase. The latter is due to the additional crossover operations.

The comparison between different crossover rates illustrates that the two crossover rates 0.5 and 0.7 have an acceptable performance in terms of both solution gap and computation time.

Consequently, increasing or decreasing each parameter is based on the managers emphasizing the accuracy or efficiency of the algorithm. For example, if the manager sets acceptable error rate in advance, the population size of $60 \mathrm{can}$ be used in all test problems with maximum error rate of $4.5 \%$. 


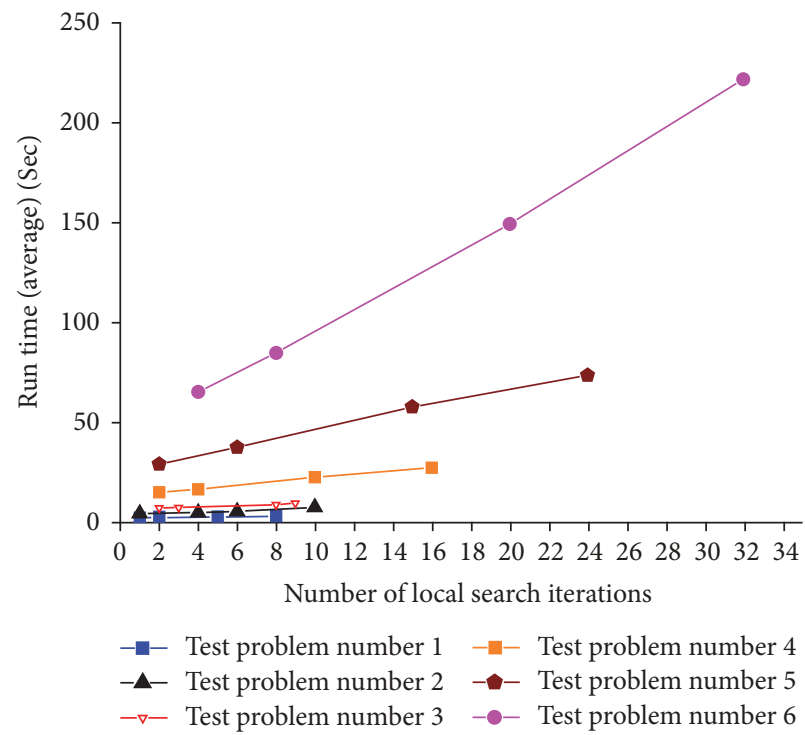

(a) Runtime of the proposed MA for different number of local search iterations

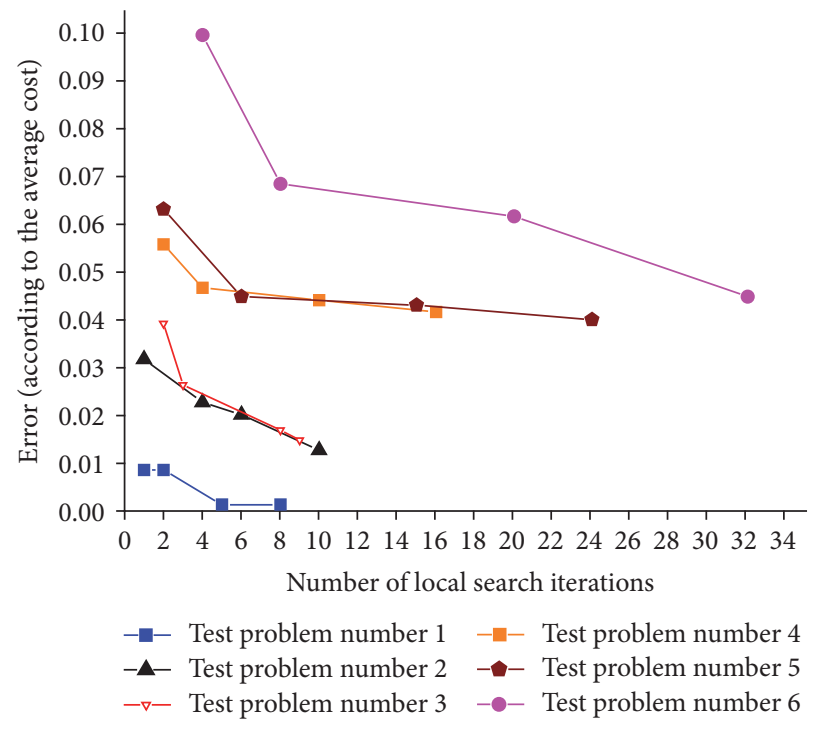

(b) Gap of the proposed MA for different number of local search iterations

FIGURE 11: Effect of different number of local search iterations.

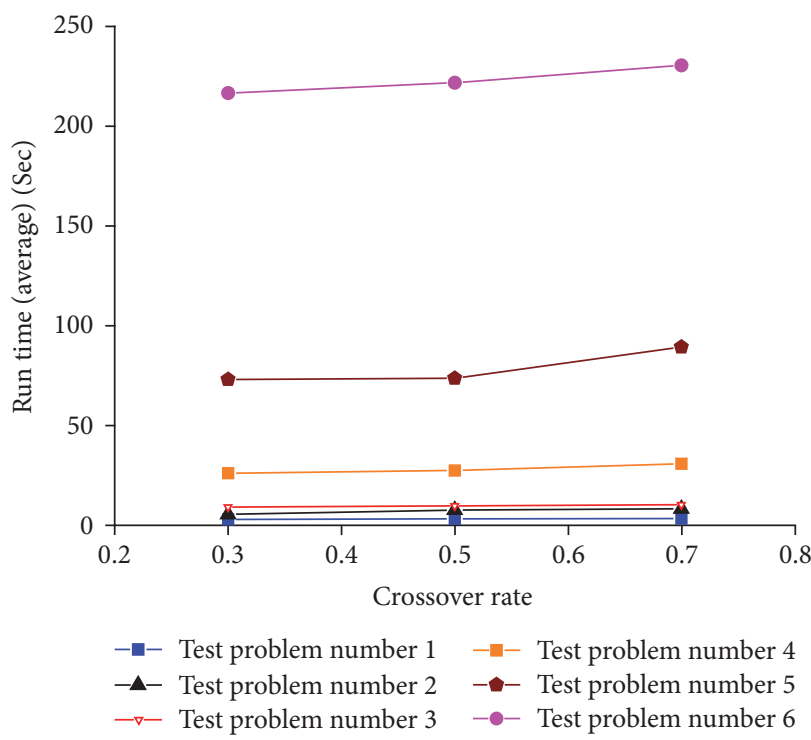

(a) Runtime of the proposed MA for different crossover rates

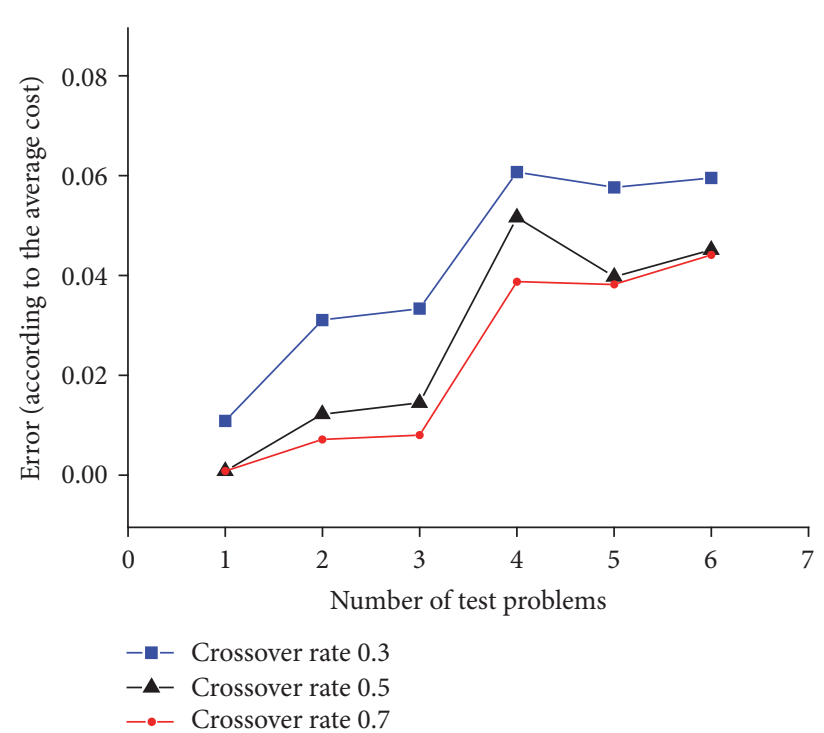

(b) Gap of the proposed MA for different crossover rates

FIGURE 12: Effect of different crossover rates.

On the other hand, by sacrificing efficiency of the algorithm more accurate results and lower error rates can be obtained by variable population size approach.

\section{Conclusions and Future Studies}

From our experiments, we have observed that large population sizes, large number of local search iterations, and high crossover rates can improve the solution. Yet, the respective computation time is very high. We analyzed the trade-off between error percentage and computation time to identify suitable parameters. If predefined acceptable error rate and computation time are given $[8,17]$, the respective parameters can be determined. In summary, the proposed algorithm has demonstrated its performance regarding both efficiency and accuracy.

In the future, we plan to apply Taguchi analysis to determine the best parameter values as a general suggestion for the proposed method.

\section{Conflicts of Interest}

The authors declare that there are no conflicts of interest regarding the publication of this paper. 


\section{Acknowledgments}

The authors would like to appreciate the International Graduate School (IGS) of Bremen University for support, help, and advice as well as the Deutscher Akademischer Austauschdienst (DAAD) for financial support of this research under the GSSP Programme of the IGS.

\section{References}

[1] A. Amiri, "Designing a distribution network in a supply chain system: formulation and efficient solution procedure," European Journal of Operational Research, vol. 171, no. 2, pp. 567-576, 2006.

[2] A. Dasci and V. Verter, "A continuous model for productiondistribution system design," European Journal of Operational Research, vol. 129, no. 2, pp. 287-298, 2001.

[3] O. Listeş and R. Dekker, "A stochastic approach to a case study for product recovery network design," European Journal of Operational Research, vol. 160, no. 1, pp. 268-287, 2005.

[4] J. Q. F. Neto, G. Walther, J. Bloemhof, J. A. E. E. Van Nunen, and T. Spengler, "From closed-loop to sustainable supply chains: The WEEE case," International Journal of Production Research, vol. 48, no. 15, pp. 4463-4481, 2010.

[5] L. M. Meade, J. Sarkis, and A. Presley, "The theory and practice to Reverse Logistics," International Journal of Logistics Systems and Management, vol. 3, no. 1, pp. 56-84, 2007.

[6] H. Uster, G. Easwaran, E. Akali, and S. Cetinkaya, "Benders decomposition with alternative multiple cuts for a multiproduct closed-loop supply chain network design model," Naval Research Logistics (NRL), vol. 54, no. 8, pp. 890-907, 2007.

[7] S. A. Alumur, S. Nickel, F. Saldanha-da-Gama, and V. Verter, "Multi-period reverse logistics network design," European Journal of Operational Research, vol. 220, no. 1, pp. 67-78, 2012.

[8] D.-H. Lee and M. Dong, "A heuristic approach to logistics network design for end-of-lease computer products recovery," Transportation Research Part E: Logistics and Transportation Review, vol. 44, no. 3, pp. 455-474, 2008.

[9] M. S. Pishvaee, R. Z. Farahani, and W. Dullaert, "A memetic algorithm for bi-objective integrated forward/reverse logistics network design," Computers \& Operations Research, vol. 37, no. 6, pp. 1100-1112, 2010.

[10] W. Klibi, A. Martel, and A. Guitouni, "The design of robust value-creating supply chain networks: a critical review," European Journal of Operational Research, vol. 203, no. 2, pp. 283293, 2010.

[11] F. Altiparmak, M. Gen, L. Lin, and T. Paksoy, "A genetic algorithm approach for multi-objective optimization of supply chain networks," Computers \& Industrial Engineering, vol. 51, no. 1, pp. 196-215, 2006.

[12] F. Du and G. W. Evans, "A bi-objective reverse logistics network analysis for post-sale service," Computers \& Operations Research, vol. 35, no. 8, pp. 2617-2634, 2008.

[13] W.-C. Yeh, "A hybrid heuristic algorithm for the multistage supply chain network problem," The International Journal of Advanced Manufacturing Technology, vol. 26, no. 5-6, pp. 675685, 2005.

[14] V. Jayaraman, R. A. Patterson, and E. Rolland, "The design of reverse distribution networks: models and solution procedures," European Journal of Operational Research, vol. 150, no. 1, pp. 128-149, 2003.
[15] H. Min and H. Ko, "The dynamic design of a reverse logistics network from the perspective of third-party logistics service providers," International Journal of Production Economics, vol. 113, no. 1, pp. 176-192, 2008.

[16] J. B. Jo, L. Yinzhen, and M. Gen, "Nonlinear fixed charge transportation problem by spanning tree-based genetic algorithm," Computers \& Industrial Engineering, vol. 53, no. 2, pp. 290-298, 2007.

[17] H. F. Wang and H. W. Hsu, "A closed-loop logistic model with a spanning-tree based genetic algorithm," Computers \& Operations Research, vol. 37, no. 2, pp. 376-389, 2010.

[18] G. Kannan, S. Pokharel, and P. S. Kumar, "A hybrid approach using ISM and fuzzy TOPSIS for the selection of reverse logistics provider," Resources, Conservation \& Recycling, vol. 54, no. 1, pp. 28-36, 2009.

[19] B. Dengiz, F. Altiparmak, and A. E. Smith, "Local search genetic algorithm for optimal design of reliable networks," IEEE Transactions on Evolutionary Computation, vol. 1, no. 3, pp. 179188, 1997.

[20] M. S. Pishvaee, K. Kianfar, and B. Karimi, "Reverse logistics network design using simulated annealing," The International Journal of Advanced Manufacturing Technology, vol. 47, no. 1-4, pp. 269-281, 2010.

[21] V. Jayaraman and A. Ross, "A simulated annealing methodology to distribution network design and management," European Journal of Operational Research, vol. 144, no. 3, pp. 629-645, 2003.

[22] C. S. Sung and S. H. Song, "Integrated service network design for a cross-docking supply chain network," Journal of the Operational Research Society, vol. 54, no. 12, pp. 1283-1295, 2003.

[23] M. T. Melo, S. Nickel, and F. Saldanha-da-Gama, "Facility location and supply chain management-a review," European Journal of Operational Research, vol. 196, no. 2, pp. 401-412, 2009.

[24] A. Syarif, Y. Yun, and M. Gen, "Study on multi-stage logistic chain network: a spanning tree-based genetic algorithm approach," Computers \& Industrial Engineering, vol. 43, no. 1-2, pp. 299-314, 2002.

[25] E. Behmanesh and J. Pannek, "A memetic algorithm with extended random path encoding for a closed-loop supply chain model with flexible delivery," Logistics Research, vol. 9, no. 1, article no. 22, 2016.

[26] M. Gen, F. Altiparmak, and L. Lin, "A genetic algorithm for twostage transportation problem using priority-based encoding," OR Spectrum, vol. 28, no. 3, pp. 337-354, 2006.

[27] H. Khorshidian, N. Javadian, M. Zandieh, J. Rezaeian, and K. Rahmani, "A genetic algorithm for JIT single machine scheduling with preemption and machine idle time," Expert Systems with Applications, vol. 38, no. 7, pp. 7911-7918, 2011.

[28] M. Zandieh, M. Amiri, B. Vahdani, and R. Soltani, "A robust parameter design for multi-response problems," Journal of Computational and Applied Mathematics, vol. 230, no. 2, pp. 463-476, 2009.

[29] M. S. Pishvaee, F. Jolai, and J. Razmi, "A stochastic optimization model for integrated forward/reverse logistics network design," Journal of Manufacturing Systems, vol. 28, no. 4, pp. 107-114, 2009.

[30] B. Beamon, "Designing the green supply chain," Logistics Information Management, vol. 12, no. 4, pp. 332-342, 1999.

[31] M. Gen and R. Cheng, Genetic Algorithms and Engineering Design, John Wiley \& Sons, New York, NY, USA, 1997. 
[32] J. Gottlieb and L. Paulmann, "Genetic algorithms for the fixed charge transportation problem," in IEEE World Congress on evolutionary computation, I. C. Inn, Ed., pp. 330-335, 1998.

[33] E. Behmanesh and J. Pannek, "Modeling and random pathbased direct encoding for a closed loop supply chain model with flexible delivery paths," IFAC-PapersOnLine, vol. 49, no. 2, pp. 78-83, 2016.

[34] M. Boudia and C. Prins, "A memetic algorithm with dynamic population management for an integrated production-distribution problem," European Journal of Operational Research, vol. 195, no. 3, pp. 703-715, 2009.

[35] R. T. Moghaddam, N. Safaei, and F. Sassani, "A memetic algorithm for the flexible flow line scheduling problem with processor blocking," Computers \& Operations Research, vol. 36, no. 2, pp. 402-414, 2009.

[36] S. Kim, A. E. Smith, and J. Lee, "A memetic algorithm for channel assignment in wireless FDMA systems," Computers \& Operations Research, vol. 34, no. 6, pp. 1842-1856, 2007.

[37] T. Y. ElMekkawy and S. Liu, "A new memetic algorithm for optimizing the partitioning problem of tandem AGV systems," International Journal of Production Economics, vol. 118, no. 2, pp. 508-520, 2009.

[38] M. Safe, J. Carballido, I. Ponzoni, and N. Brignole, "On stopping criteria for genetic algorithms," in Advances in Artificial Intelligence SBIA, I. C. Inn, Ed., pp. 330-335, 2004.

[39] A. P. Piotrowski, "Review of Differential Evolution population size," Swarm and Evolutionary Computation, vol. 32, pp. 1-24, 2017. 


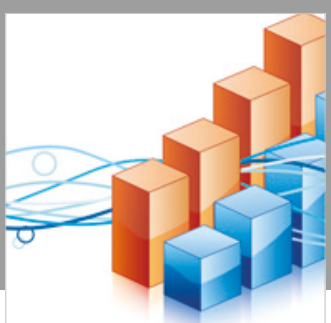

Advances in

Operations Research

\section{-n-m}
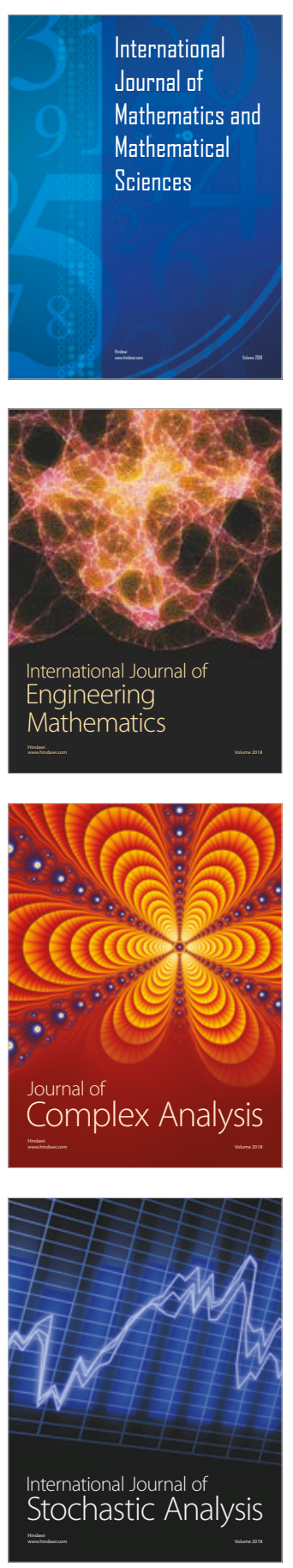
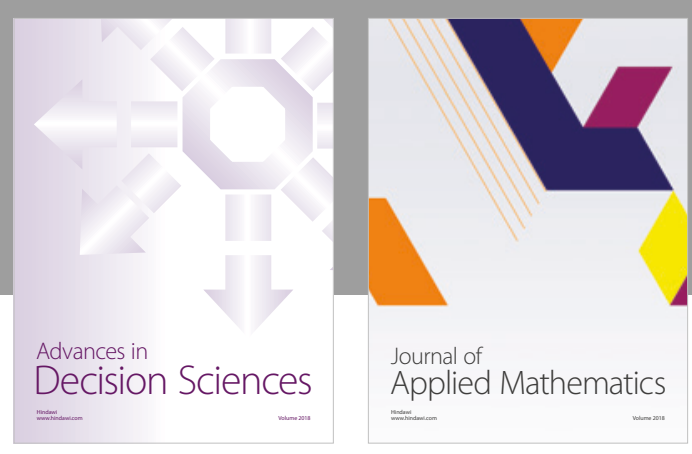

Journal of

Applied Mathematics
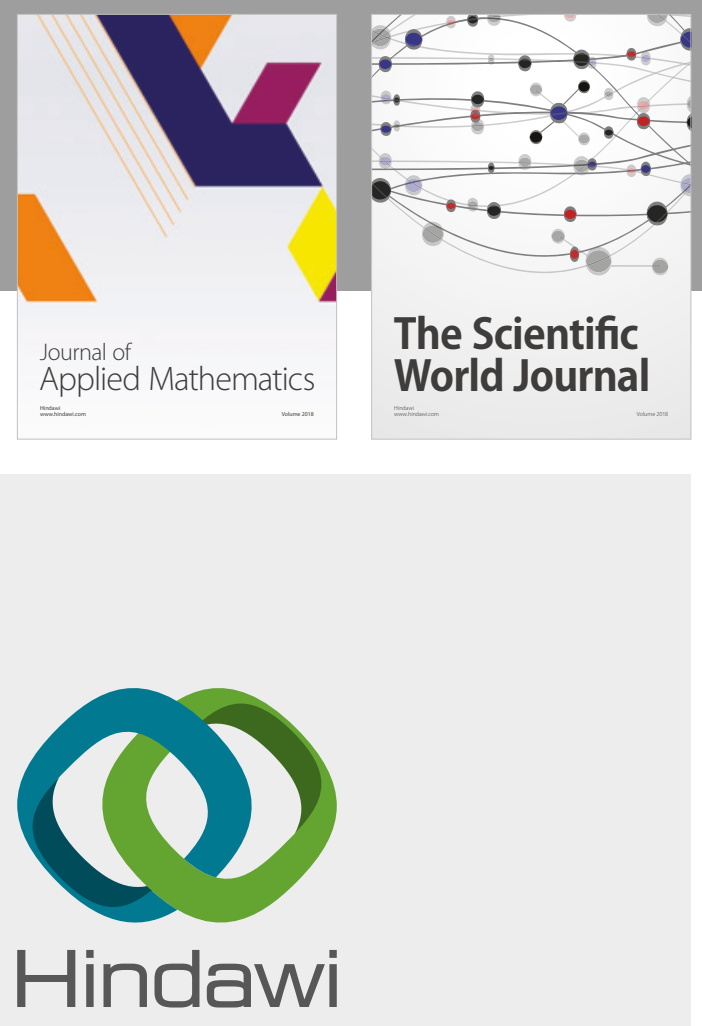

Submit your manuscripts at

www.hindawi.com

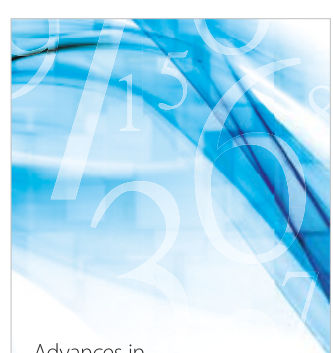

Advances in
Numerical Analysis
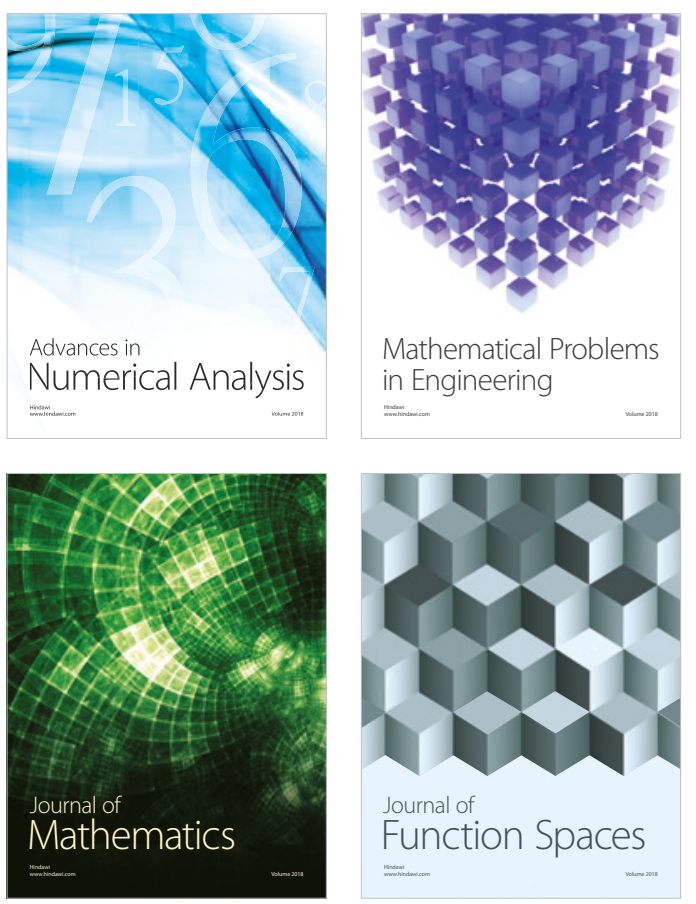

Mathematical Problems in Engineering

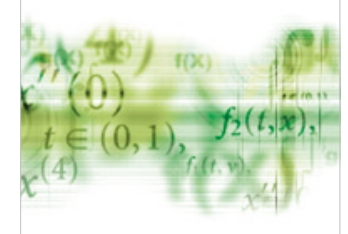

International Journal of

Differential Equations

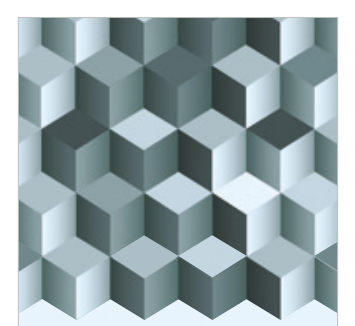

Journal of

Function Spaces

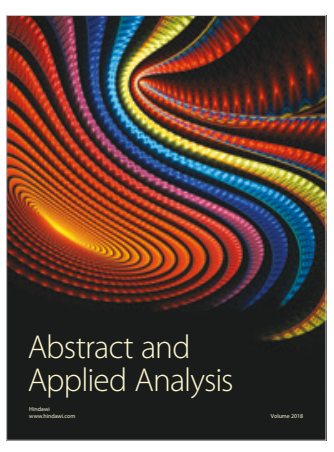

The Scientific

World Journal

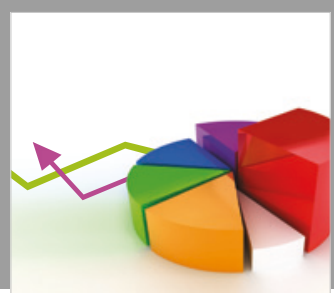

Journal of

Probability and Statistics
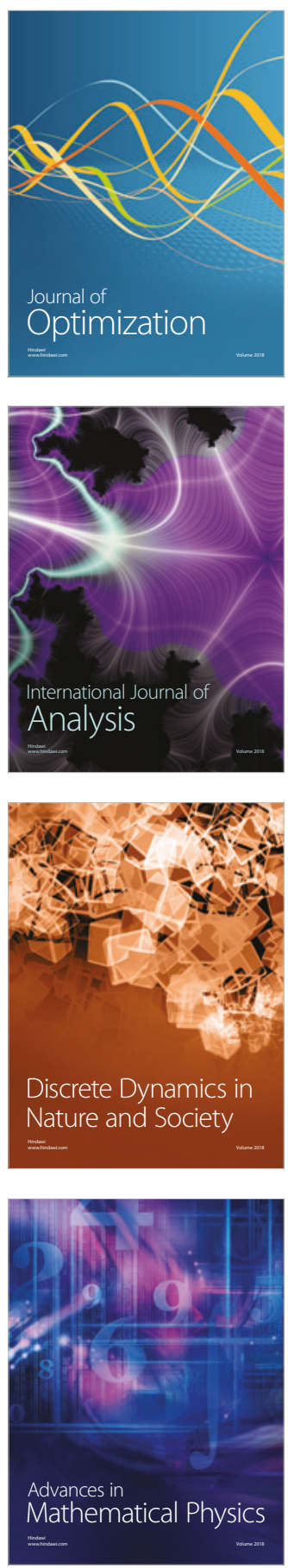